\title{
The impact of graduated compression stockings on calf-vein deformation and blood velocity in patients awaiting total knee arthroplasty
}

Zaikai Zhuang ${ }^{1 \dagger}$, Yexian Wang ${ }^{1,2+}$, Yao Yao ${ }^{1,2}$, Ying Shen ${ }^{1,2}$, Dongyang Chen ${ }^{1,2^{*}}$ and Qing Jiang ${ }^{1,2^{*}}$

\begin{abstract}
Objectives: This study was designed to explore venous deformation of the lower extremities and the changes in venous hemodynamics in supine position before and after wearing graduated elastic stockings in patients awaiting total knee arthroplasty (TKA).

Method: The leg veins of 21 elderly patients awaiting TKA were imaged in the supine position with and without knee-length graduated compression stockings (GCS) according to a fixed protocol. Measured parameters including the lateromedial (LM) diameter, anteroposterior (AP) diameter, and cross-sectional area (CSA) of the great saphenous vein (GSV), gastrocnemius vein (GV), soleus vein (SV), posterior tibial vein (PTV), fibular vein (FV), and anterior tibial vein (ATV). In addition, the mean and maximum velocities of the popliteal vein (PV) and superficial femoral vein (FSV) were measured.

Results: GCS-related compression was observed for all the measured veins. Maximal reduction was observed for the GV and SV, whereas the GSV exhibited the lowest degree of GCS-related compression. The mean cross-sectional area reduction values associated with GCS were $33.1 \pm 41.2 \%$ for the GSV, $94.8 \pm 11.1 \%$ for the GV, and $85.6 \pm$ $20.3 \%$ for the SV, while the mean reduction of anteroposterior diameter was $18.1 \pm 34.5 \%$ for the GSV, $89.0 \pm 22.5 \%$ for the GV, and $72.9 \pm 35.1 \%$ for the SV, and the mean reduction of the lateromedial diameter was $25.9 \pm 36.4 \%$ for the GSV, $89.6 \pm 19.6 \%$ for the GV, $78.2 \pm 28.3 \%$ for the SV. No significant GCS-related changes in blood velocity in the superficial femoral veins or popliteal veins were detected.

Conclusions: For elderly patients awaiting TKA, knee-length GCS can significantly reduce calf vein dilation while at rest in the supine position, with the greatest reductions being observed for the soleus and gastrocnemius veins. These data might help provide a theoretical basis for the GCS in reducing incidence of deep vein thrombosis in patients undergoing TKA.
\end{abstract}

Keywords: Graduated compression stockings, Vein diameter, Blood velocity, Venous thrombosis, Thromboprophylaxis

\footnotetext{
* Correspondence: 28617621@qq.com; qingj@nju.edu.cn

'Zaikai Zhuang and Yexian Wang contributed equally to this work and could be regarded as the first authors.

'Nanjing Drum Tower Hospital Clinical College of Nanjing Medical University, Nanjing 210008, People's Republic of China

Full list of author information is available at the end of the article
}

C C The Author(s). 2021 Open Access This article is licensed under a Creative Commons Attribution 4.0 International License, which permits use, sharing, adaptation, distribution and reproduction in any medium or format, as long as you give appropriate credit to the original author(s) and the source, provide a link to the Creative Commons licence, and indicate if changes were made. The images or other third party material in this article are included in the article's Creative Commons licence, unless indicated otherwise in a credit line to the material. If material is not included in the article's Creative Commons licence and your intended use is not permitted by statutory regulation or exceeds the permitted use, you will need to obtain permission directly from the copyright holder. To view a copy of this licence, visit http://creativecommons.org/licenses/by/4.0/. The Creative Commons Public Domain Dedication waiver (http://creativecommons.org/publicdomain/zero/1.0/) applies to the data made available in this article, unless otherwise stated in a credit line to the data. 


\section{Introduction}

Deep vein thrombosis (DVT) is defined as the occurrence of a blood clot within a deep vein, most often in the legs. Thrombus formation primarily occurs in the context of immobilization, the rate of DVT following knee arthroplasty ranges from 41 to $85 \%$ without thromboprophylaxis $[1,2]$. In-hospital mortality rates for patients suffering from venous thrombosis are roughly $1.1 \%$, and these patients experience mortality rates of $9.4 \%$ over a 6-month follow-up period [3]. Some prior reports have suggested that up to $50 \%$ of patients may develop post-thrombotic syndrome (PTS) following DVT, potentially reducing their disease-specific quality of life $[4,5]$. Preventing DVT following knee arthroplasty is thus essential.

The intermuscular vein thrombosis is the most common distal lower limb thrombus [6]. And we and others have previously reported that soleus vein (SV) dilatation is an independent predictor of DVT risk following major orthopedic surgery $[7,8]$.

Mechanical thromboprophylaxis is most commonly achieved by using graduated compression stockings (GCS), as wearing GSC has been shown to significantly reduce the risk of DVT in orthopedic surgery patients [9]. Some reviews have found that GCS may decrease DVT incidence by reducing venous diameters and increasing rates of venous blood flow $[10,11]$. So we would like to observe whether GCS can eliminate the risk factor for intermuscular vein dilation in patients undergoing TKA.

This study was designed to analyze lower extremity venous deformation before and after wearing graduated elastic stockings in patients awaiting TKA, with a focus on the soleus and gastrocnemius veins. The overall goal of this study was to help provide a theoretical basis for the GCS in reducing incidence of deep vein thrombosis in patients undergoing TKA.

\section{Materials and methods}

All methods were carried out in accordance with the Declaration of Helsinki.

The hospital Ethics Committee approved the present study, and all patients provided informed consent. In total, 21 patients awaiting TKA were analyzed. Patients were excluded from this study if they presented with comorbidities including severe peripheral neuropathy, severe lower extremity arterial disease, severe lower limb deformities, thrombosis, sensory impairment, and visible varicose veins. Patients were additionally excluded if they were excessively obese $(B M I \geq 40)$, excessively underweight (BMI $<18.5)$, or had undergone venous surgical procedures.

\section{Experimental protocols}

All measurements were made in a room with a comfortable environment. Patients were instructed to lay in the supine position with both of their legs being exposed. A pillow (8 cm in height) was placed $\sim 5 \mathrm{~cm}$ above the popliteal fossa to allow for ease of measurement. To ensure that the same vein segment in the same position was measured, three marking lines were made on the skin with a non-water-soluble black ink pen. Lines were located at the midpoint of the thigh, the knee joint space, and the midpoint of the calf.

Patients were allowed to rest for 15 min until their blood pressure and heart rate stabilized, after which baseline measurements of the lower limb were made. For these measurements, the great saphenous vein, anterior tibial vein, posterior tibial vein, and fibular vein were assessed at the midpoint of the calf, the popliteal vein was assessed at the knee joint space, and the superficial femoral vein was assessed at the midpoint of the thigh. The gastrocnemius vein and soleus vein were measured at the largest diameter of the vein via ultrasound, with the distance between the vein and the probe being recorded and the skin being marked with a pen to ensure that subsequent scans were made at the same location. Prior studies have utilized vein diameter as a metric for characterizing decreases in venous crosssectional area, which would suggest that the vessel cross-section remained roughly circular. However, we found that vein cross-sectional geometry was roughly elliptical before and after compression. As such, we measured the lateromedial diameter, anteroposterior diameter, and cross-sectional area of each vein to more accurately reflect venous deformation. The mean and maximum blood velocity values for the superficial femoral vein and the popliteal vein were additionally recorded.

After patients put on the compressive stockings, they were allowed to rest for an additional $15 \mathrm{~min}$ prior to analysis. To ensure appropriate visibility of the veins across the stockings, a sufficient amount of gel was applied such that the underlying tissue was wet. A new set of duplex measurements was then made for each vein through the compressive stockings. Superficial femoral vein and popliteal vein scans were taken above the proximal edge of the stocking. Care was taken to avoid applying additional pressure to the limb with the transducer, as this had the potential to alter the venous diameter.

\section{Compression stockings}

Knee-length gradient elastic compression stocking size was selected using the measuring chart recommended by the manufacturer (Medical Supplies Pty Ltd, Haoshide, China). The stockings used for this study were of an elastic knit with $16-22 \mathrm{mmHg}$ of compression 
pressure (compression class I). Studies have reported that with respect to postoperative thromboprophylaxis, there were no differences between the knee-length, lowpressure GCS and GCS of other sizes or pressures, whereas patients report feeling more comfortable when wearing knee-length, low-pressure GCS [12].

\section{Doppler ultrasound measurements}

A Sonosite M-Turbo ultrasound system was used for all 2D transverse scans of the veins. After appropriate scans were complete, images were frozen and the anteroposterior diameter, lateromedial diameter, and cross-sectional area of each vein were measured (Fig. 1). Longitudinal scans were obtained for the superficial femoral vein and the popliteal vein. The Doppler sample volume cursor was placed in the center of this vein, and the mean blood velocity and maximum blood velocity were calculated (Fig. 2). Mean values for these three measurements were made.

\section{Statistical analysis}

SPSS v. 26 (IBM Corp., Armonk, NY, USA) was used for all statistical testing. Data are given as means with standard deviations (SD), and were analyzed via Wilcoxon rank-sum test. $P<0.05$ was the significance threshold for this study.

\section{Results}

In total, 21 patients were included in the present study with a mean age of $64 \pm 7$ years (range: 54-76 years). The mean body mass index (BMI) of these patients was $26.6 \pm 3.4 \mathrm{~kg} / \mathrm{m}^{2}$ (range: $21.3-35.1 \mathrm{~kg} / \mathrm{m}^{2}$ ), and $17 / 21 \mathrm{pa}$ tients were female.

Wearing GCS was associated with the compression of most analyzed veins including the GSV, GV, SV, PTV, FV, and ATV. Maximal reduction was observed for the GV and SV, whereas the GSV exhibited the lowest degree of reduction. Gastrocnemius veins were closed in 16 of these 21 patients, while soleus veins were closed in 12 of these 21 patients in the context of GCS-mediated compression. Representative images of GV and SV deformation are shown in Fig. 3.
Cross-sectional areas and diameters of the calf muscle veins in these patients are shown in Table 1 . The GV exhibited mean uncompressed and compressed crosssectional area values of $21.5 \pm 25.1 \mathrm{~mm}^{2}$ and $2.4 \pm 6.2$ $\mathrm{mm}^{2}$, respectively $(P<0.001)$. Similarly, GCS were associated with a significant reduction in GV diameter from $5.1 \pm 3.1 \mathrm{~mm}$ to $0.9 \pm 2.1 \mathrm{~mm}$ (AP; $P<0.001$ ) and $4.4 \pm$ $2.3 \mathrm{~mm}$ to $0.7 \pm 1.6$ (LM; $P<0.001$ ). The SV also exhibited significant GCS-related reductions in cross-sectional area in these patients $\left(40.3 \pm 25.0 \mathrm{~mm}^{2}\right.$ vs. $5.0 \pm 7.3$ $\left.\mathrm{mm}^{2} ; P<0.001\right)$. GCS additionally decreased the diameter of the SV from $8.1 \pm 2.5 \mathrm{~mm}$ to $2.1 \pm 2.7 \mathrm{~mm}$ (AP; $P$ $<0.001)$ and $5.8 \pm 2.0 \mathrm{~mm}$ to $1.3 \pm 1.7$ (LM; $P<0.001$ ). The mean cross-sectional area reductions for the GV and SV were $94.8 \pm 11.1 \%$ and $85.6 \pm 20.3 \%$, respectively.

Cross-sectional area values and diameters for all deep veins measured in the present study are shown in Table 2. A significant GCS-related reduction in the cross-sectional area of the ATV was observed in these patients $\left(4.2 \pm 2.4 \mathrm{~mm}^{2}\right.$ to $\left.1.1 \pm 1.0 \mathrm{~mm}^{2} ; P<0.001\right)$, and this coincided with a compression-mediated decrease in ATV diameter from $1.9 \pm 0.6 \mathrm{~mm}$ to $0.8 \pm 0.7 \mathrm{~mm}$ (AP; $P<0.001)$ and $3.0 \pm 0.9 \mathrm{~mm}$ to $1.0 \pm 0.9$ (LM; $P<0.001$ ). The cross-sectional area of the PTV similarly differed in a GCS-related manner $\left(15.4 \pm 12.3 \mathrm{~mm}^{2}\right.$ vs. $4.8 \pm 3.7$ $\left.\mathrm{mm}^{2} ; P<0.001\right)$, and the PTV diameter declined from $4.3 \pm 2.0 \mathrm{~mm}$ to $2.5 \pm 0.8 \mathrm{~mm}$ (AP; $P<0.001$ ) and $3.9 \pm$ $1.6 \mathrm{~mm}$ to $2.3 \pm 1.3$ (LM; $P<0.001$ ) when patients wore GCS. GCS application reduced the cross-sectional area of the FV in these patients from $44.0 \pm 19.2 \mathrm{~mm}^{2}$ to $14.2 \pm 11.4 \mathrm{~mm}^{2}(P<0.001)$ and reduced the diameter of the FV from $8.6 \pm 2.3 \mathrm{~mm}$ to $3.9 \pm 2.3 \mathrm{~mm}$ (AP; $P<$ $0.001)$ and $6.2 \pm 1.7 \mathrm{~mm}$ to $3.4 \pm 2.6(\mathrm{LM} ; P=0.001)$. The mean CSA reductions for the ATV, PTV, and FV were $69.5 \pm 29.8 \%, \quad 59.0 \pm 28.2 \%, \quad$ and $\quad 65.2 \pm 30.5 \%$, respectively.

Cross-sectional area and diameter values for superficial veins analyzed in the present study are shown in Table 3. GCS application was associated with a significant reduction in the GSV cross-sectional area from $5.1 \pm 3.4 \mathrm{~mm}^{2}$
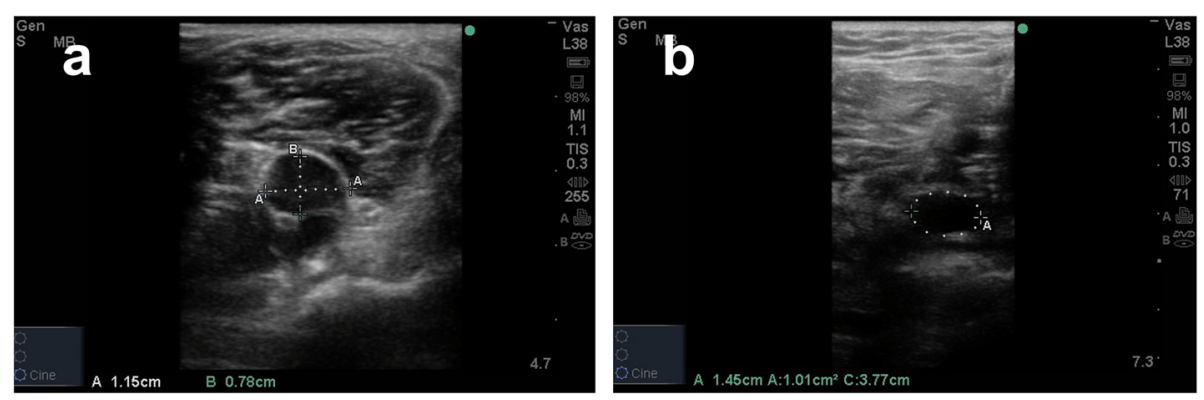

Fig. 1 a Line A represents the lateromedial diameter of the vein and line B represents the anteroposterior diameter of the vein. $\mathbf{b}$ The crosssectional area of the vein 


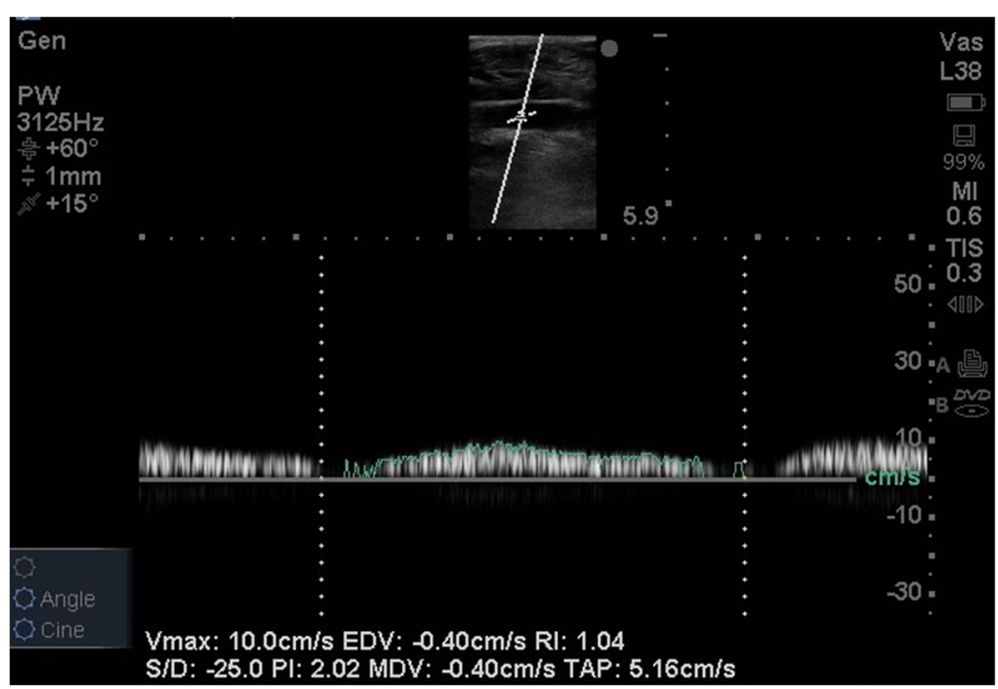

Fig. 2 The Doppler sample volume cursor was placed in the center of the vein, and mean blood velocity and maximum blood velocity were calculated

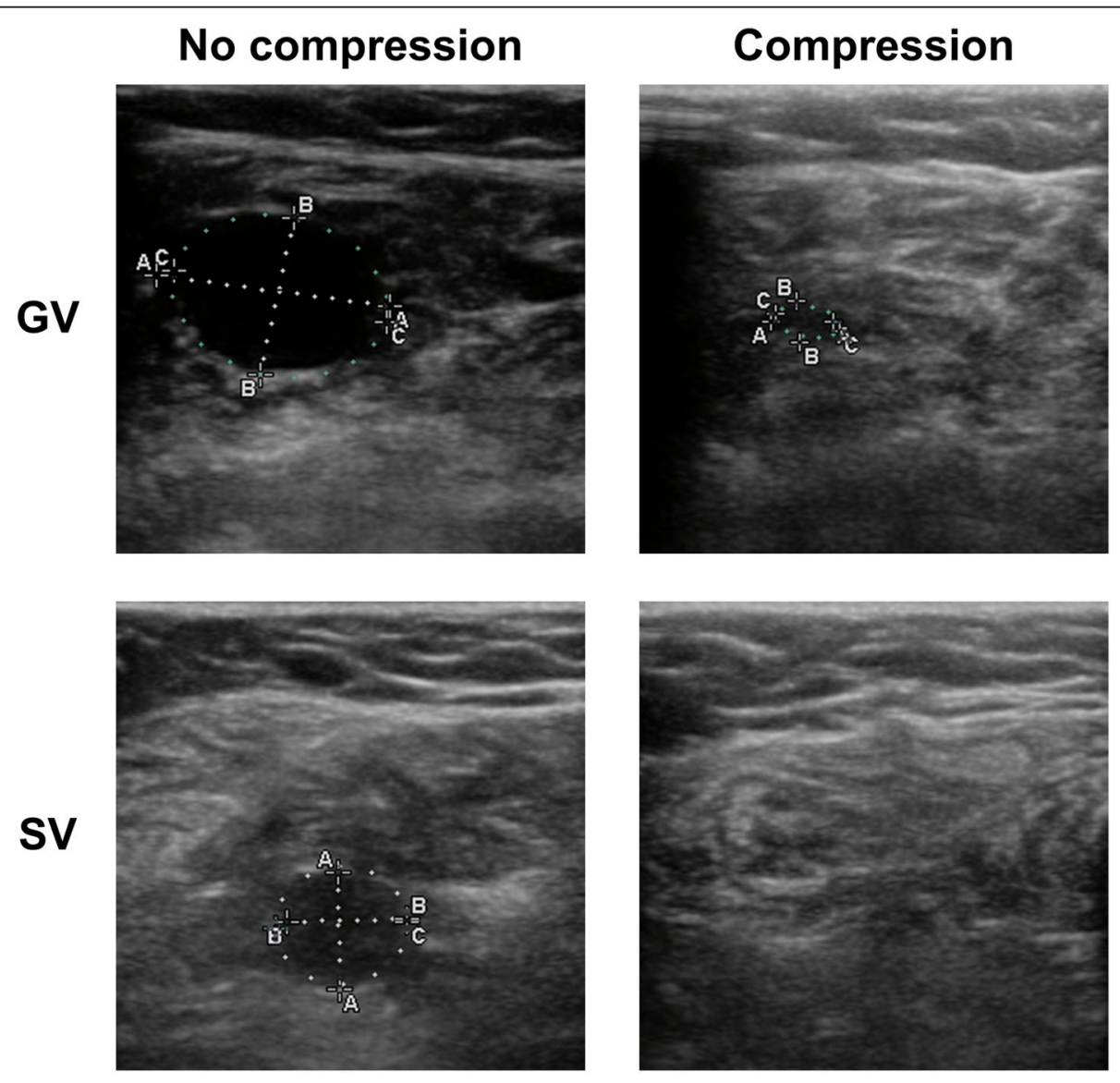

Fig. 3 Duplex sonography imaging of gastrocnemius veins and soleus veins, with and without compression. $\mathbf{A}=$ lateromedial diameter, $\mathbf{B}=$ anteroposterior diameter 
Table 1 The deformation of the calf muscle veins in compressed states

\begin{tabular}{lcccc}
\hline & Uncompressed & Compressed & $P$-value & \% Reduction \\
\hline GV CSA $\left(\mathrm{mm}^{2}\right)$ & $21.5 \pm 25.1$ & $2.4 \pm 6.2$ & $<0.001$ & $94.8 \pm 11.1$ \\
GV diameter AP $(\mathrm{mm})$ & $5.1 \pm 3.1$ & $0.9 \pm 2.1$ & $<0.001$ & $89.0 \pm 22.5$ \\
GV diameter LM (mm) & $4.4 \pm 2.3$ & $0.7 \pm 1.6$ & $<0.001$ & $89.6 \pm 19.6$ \\
SV CSA $\left(\mathrm{mm}^{2}\right)$ & $40.3 \pm 25.0$ & $5.0 \pm 7.3$ & $<0.001$ & $85.6 \pm 20.3$ \\
SV diameter AP $(\mathrm{mm})$ & $8.1 \pm 2.5$ & $2.1 \pm 2.7$ & $<0.001$ & $72.9 \pm 35.1$ \\
SV diameter LM (mm) & $5.8 \pm 2.0$ & $1.3 \pm 1.7$ & $<0.001$ & $78.2 \pm 28.3$ \\
\hline
\end{tabular}

Data are means \pm SD

GV gastrocnemius vein, SV soleus vein, CSA cross sectional area, AP anteroposterior, $L M$ lateromedial

to $3.2 \pm 2.6 \mathrm{~mm}^{2},(P=0.002)$ and in GSV diameter from $2.8 \pm 1.0 \mathrm{~mm}$ to $2.3 \pm 1.2 \mathrm{~mm}$ (AP; $P<0.001$ ) and $2.1 \pm$ $0.8 \mathrm{~mm}$ to $1.4 \pm 0.6$ (LM; $P=0.003$ ). The mean GCSrelated cross-sectional area reduction for the GSV was $33.1 \pm 41.2 \%$ in these patients.

The peak blood velocity in the femoral vein of all 21 patients without GCS was $16.1 \pm 3.9 \mathrm{~cm} / \mathrm{s}$, and it did not significantly increase in these patients with GCS (16.6 \pm $4.3 \mathrm{~cm} / \mathrm{s} ; P=0.339)$. Similarly, no GCS-related increases in mean blood velocity values in the femoral vein were observed $(9.9 \pm 3.7 \mathrm{~cm} / \mathrm{s}$ vs. $10.7 \pm 4.2 \mathrm{~cm} / \mathrm{s} ; P=0.068)$ (Table 4).

The peak blood velocity in the popliteal vein of all 21 patients without GCS was $9.5 \pm 3.0 \mathrm{~cm} / \mathrm{s}$, and it did not significantly increase in these patients with GCS $(10.1 \pm$ $3.5 \mathrm{~cm} / \mathrm{s} ; P=0.227$ ). Similarly, no GCS-related increases in mean blood velocity values in the popliteal vein were observed $(5.1 \pm 2.0 \mathrm{~cm} / \mathrm{s}$ vs. $5.3 \pm 2.5 \mathrm{~cm} / \mathrm{s} ; \quad P=0.414)$ (Table 4).

\section{Discussion}

The present study was designed to explore the GCSrelated deformation of lower extremity veins and associated hemodynamic changes in patients awaiting TKA in the supine position. Through these analyses, we found that GCS were able to significantly compress most measured veins including the GSV, GV, SV, PTV, FV, and
ATV, with the effect being most pronounced for the GV and SV. No significant changes in blood velocity in the superficial femoral or popliteal veins were observed.

Our results are consistent with those of multiple prior studies, which have demonstrated that GCS can significantly compress calf muscle veins. However, the percentage of mean vein reduction in this study was higher than in previous reports. Lord et al. [13] observe effective compression of both superficial and deep veins by GCS when patients were in the supine position, with this reduction being superior for the calf muscle veins. The mean internal diameter of the uncompressed and compressed SV in their study was $2.20 \mathrm{~mm}$ and $0.86 \mathrm{~mm}$, respectively, with a $60.9 \%$ mean reduction in the context of GCS-mediated compression. Arcelus et al. [14] found that the use of compressive stockings was associated with a mean reduction in the medial GV cross-sectional area of $54.5 \%$ with a corresponding $30 \%$ reduction in the lateral gastrocnemius vein cross-sectional area. Smith et al. [15] analyzed patients undergoing abdominal or neck surgery and found that the median GV diameter fell significantly from $2.6 \mathrm{~mm}$ to $1.6 \mathrm{~mm}$ following GCS application during surgery. Furthermore, Jeanneret et al. [16] determined that GCS were sufficient to significantly compress the $\mathrm{GV}$ as measured through compression stockings in the prone position. In the present report, we found that some calf muscle veins were not closed in

Table 2 The deformation of the deep veins in compressed states

\begin{tabular}{lcccc}
\hline & Uncompressed & Compressed & P-value & \% Reduction \\
\hline ATV CSA $\left(\mathrm{mm}^{2}\right)$ & $4.2 \pm 2.4$ & $1.1 \pm 1.0$ & $<0.001$ & $69.5 \pm 29.8$ \\
ATV diameter AP $(\mathrm{mm})$ & $1.9 \pm 0.6$ & $0.8 \pm 0.7$ & $<0.001$ & $57.6 \pm 39.2$ \\
ATV diameter LM (mm) & $3.0 \pm 0.9$ & $1.0 \pm 0.9$ & $<0.001$ & $62.7 \pm 33.3$ \\
PTV CSA $\left(\mathrm{mm}^{2}\right)$ & $15.4 \pm 12.3$ & $4.8 \pm 3.7$ & $<0.001$ & $59.0 \pm 28.2$ \\
PTV diameter AP (mm) & $4.3 \pm 2.0$ & $2.5 \pm 0.8$ & $<0.001$ & $35.1 \pm 31.0$ \\
PTV diameter LM (mm) & $3.9 \pm 1.6$ & $2.3 \pm 1.3$ & $<0.001$ & $37.5 \pm 31.9$ \\
FV CSA $\left(\mathrm{mm}^{2}\right)$ & $44.0 \pm 19.2$ & $14.2 \pm 11.4$ & $<0.001$ & $65.2 \pm 30.5$ \\
FV diameter AP $(\mathrm{mm})$ & $8.6 \pm 2.3$ & $3.9 \pm 2.3$ & $<0.001$ & $50.0 \pm 35.3$ \\
FV diameter LM (mm) & $6.2 \pm 1.7$ & $3.4 \pm 2.6$ & 0.001 & $48.4 \pm 38.7$ \\
\hline
\end{tabular}

Data are means \pm SD

$A T V$ anterior tibial vein, PTV posterior tibial vein, FV fibular vein, CSA cross sectional area, $A P$ anteroposterior, $L M$ lateromedial 
Table 3 The deformation of the great saphenous vein in compressed states

\begin{tabular}{lcccc}
\hline & Uncompressed & Compressed & P-value & \% Reduction \\
\hline GSV CSA $\left(\mathrm{mm}^{2}\right)$ & $5.1 \pm 3.4$ & $3.2 \pm 2.6$ & 0.002 & $33.1 \pm 41.2$ \\
GSV diameter AP $(\mathrm{mm})$ & $2.8 \pm 1.0$ & $2.3 \pm 1.2$ & $<0.001$ & $18.1 \pm 34.5$ \\
GSV diameter LM (mm) & $2.1 \pm 0.8$ & $1.4 \pm 0.6$ & 0.003 & $25.9 \pm 36.4$ \\
\hline
\end{tabular}

Data are means \pm SD

GSV great saphenous vein, CSA cross sectional area, $A P$ anteroposterior, $L M$ lateromedial

analyzed patients. This may be attributable to the fact that we utilized class 1 elastic compression GCS with a compression pressure of $16-22 \mathrm{mmHg}$, or it may be due to differences in the locations at which these calf muscle veins were measured.

GCS have also been reported to effectively compress deep veins. For example, in their study of patients in the supine position, Lord et al. found that the PTV internal diameter fell from $2.83 \mathrm{~mm}$ without GSC to $1.69 \mathrm{~mm}$ with GCS, with the FV internal diameter similarly being reduced significantly from $3.51 \mathrm{~mm}$ to $1.92 \mathrm{~mm}$ [13]. Downie et al. [17] further found that the cross-sectional area of the PTV and FV were significantly reduced by elastic compression stockings for individuals in the prone position.

GCS can similarly suppress superficial veins, although we detected less pronounced compression of these superficial veins relative to that of the calf muscle veins and deep veins in the present study. This may be because interface pressure can drive a rise in intramuscular pressure, whereas the subcutaneous pressure is solely driven by the GCS [18]. However, Partsch et al. [19] observed nearly equivalent compression of superficial and deep veins by GCS, with 3 of 9 analyzed patients exhibiting more pronounced superficial vein compression as compared to that observed for deep veins.

Unexpectedly, no significant changes in femoral or popliteal vein blood velocity were detected as a function of GCS-mediated compression. However, this is consistent with some prior reports. For example, Giron et al. [20] and Kaori et al. [21] similarly determined that knee-length GCS had no significant impact on peak flow velocity values in the femoral or popliteal veins.
Paul et al. [22, 23] and Keith et al. [24] further determined that thigh-length GCS had no significant impact on popliteal or femoral vein blood velocity for patients in the supine position. Such findings are not, however, universal. Jamieson et al. [25], for example, determined that GCS were able to significantly increase the mean blood flow velocity of the common femoral vein in pregnant women in the immediate postnatal period. Another study conducted by Espeit et al. [26] further determined that GCS were sufficient to increase the popliteal venous blood velocity of patients in the prone position. These inconsistencies may be attributable to differences in subject posture, GCS class, GCS size, the duration of GCS use, and/or the duration of immobility among studies.

We acknowledge that there are a number of limitations to the present study. First, the sample size was relatively small. However, this factor does not affect the conclusion of the paper due to the results of 21 patients revealed high consistency and the power values we calculated have exceeded a minimum threshold of 0.8 [27]. So, we considered that our data are convincing. Second, ultrasound-based analyses can be operator-dependent, although the same trained vascular sonographer conducted all measurements in this study to minimize this form of bias. Third, we only focused on knee-length GCS and did not explore the possible efficacy of compression stockings that extend above the knee. Lastly, measurements in the present study focused solely on the supine position, and additional analyses of other body positions (including standing and sitting) would better reflect the effects of GCS-mediated compression in realworld situations.

Table 4 Blood velocity in the femoral and popliteal vein with and without knee-length GCS

\begin{tabular}{lccc}
\hline & Uncompressed & Compressed & $P$-value \\
\hline PV peak flow velocity $(\mathrm{cm} / \mathrm{sec})$ & $9.5 \pm 3.0$ & $10.1 \pm 3.5$ & 0.227 \\
PV mean flow velocity $(\mathrm{cm} / \mathrm{sec})$ & $5.1 \pm 2.0$ & $5.3 \pm 2.5$ & 0.414 \\
FSV peak flow velocity $(\mathrm{cm} / \mathrm{sec})$ & $16.1 \pm 3.9$ & $16.6 \pm 4.3$ & 0.339 \\
FSV mean flow velocity $(\mathrm{cm} / \mathrm{sec})$ & $9.9 \pm 3.7$ & $10.7 \pm 4.2$ & 0.068 \\
\hline
\end{tabular}

Data are means \pm SD

PV popliteal vein, FSV superficial femoral vein 


\section{Conclusions}

In summary, the results of this study indicated that knee-length GCS can significantly reduce calf vein dilation in elderly patients awaiting TKA while in the supine position at rest, with the greatest reductions being observed for the soleus and gastrocnemius veins. These results have the potential to help provide a theoretical basis for the GCS in reducing incidence of deep vein thrombosis in patients undergoing TKA.

\section{Abbreviations}

TKA: Total knee arthroplasty; GCS: Graduated compression stockings; LM: Lateromedial; AP: Anteroposterior; CSA: Cross-sectional area; CSA: Great saphenous vein; GV: Gastrocnemius vein; SV: Soleus vein; PTV: Posterior tibial vein; FV: Fibular vein; ATV: Anterior tibial vein; PV: Popliteal vein; FSV: Superficial femoral vein; DVT: Deep vein thrombosis; PTS: Postthrombotic syndrome; BMl: Body mass index

\section{Acknowledgements}

Thanks to all the participants who took part in this research study, which made this study possible.

\section{Authors' contributions}

ZZK, YXW, QJ and DYC conceived the study; ZZK drafted the study; ZZK, $Y X W$ and $Y Y$ recruited the participants. ZZK, YXW, YY and YS collected clinical data. ZZK and $Y Y$ were responsible for statistical analyses and tables. ZZK, YXW, QJ and DYC have primary responsibility for the final content. All authors agreed to submission. The author(s) read and approved the final manuscript.

\section{Funding}

This work was supported by Key Program of NSFC (81730067), Major Project of NSFC (81991514), Jiangsu Provincial Key Medical Center Foundation,Key project in Medical science and Technology Development of Nanjing (ZKS18020).

\section{Availability of data and materials}

The datasets used and analyzed during the current study are available from the corresponding author on reasonable request.

\section{Declarations}

\section{Ethics approval and consent to participate}

All methods were carried out in accordance with the Declaration of Helsinki. This study had been approved by the Medical Ethics Committee of Nanjing University Medical Affiliated Drum Tower Hospital. Informed consent to participate was obtained from all of the individual participants included in the study.

\section{Consent for publication}

Informed consent to publication of research was obtained from the participants included in this study.

\section{Competing interests}

The authors declare that they have no competing interests.

\section{Author details}

${ }^{1}$ Nanjing Drum Tower Hospital Clinical College of Nanjing Medical University, Nanjing 210008, People's Republic of China. ${ }^{2}$ State Key Laboratory of Pharmaceutical Biotechnology, Division of Sports Medicine and Adult Reconstructive Surgery, Department of Orthopedic Surgery, Nanjing Drum Tower Hospital, The Affiliated Hospital of Nanjing University Medical School, 321 Zhongshan Road, Nanjing 210008, Jiangsu, People's Republic of China.
Received: 29 May 2021 Accepted: 9 August 2021

Published online: 23 August 2021

\section{References}

1. Baue AE. Prevention of venous thrombosis and pulmonary embolism. JAMA 1986;256(6): 757. https://doi.org/10.1001/jama.256.6.757.

2. Geerts WH, Bergqvist D, Pineo GF, Heit JA, Samama CM, Lassen MR, et al. Prevention of venous thromboembolism: American College of Chest Physicians Evidence-Based Clinical Practice Guidelines (8th Edition). Chest. 2008;133:381S-453S. https://doi.org/10.1378/chest.08-0656.

3. Murin S, Romano PS, White RH. Comparison of outcomes after hospitalization for deep venous thrombosis or pulmonary embolism. Thromb Haemost. 2002; 88(3): 407-14. https://doi.org/10.1055/s-0037-1613230

4. Kahn K, Susan R. Determinants and time course of the postthrombotic syndrome after acute deep venous thrombosis determinants of the postthrombotic syndrome. Ann Int Med. 2008;149(10): 698. https://doi.org/1 0.7326/0003-4819-149-10-200811180-00004

5. Kahn SR, Hirsch A, Shrier I. Effect of postthrombotic syndrome on healthrelated quality of life after deep venous thrombosis. Arch Int Med. 2002; 162(10): 1144. https://doi.org/10.1001/archinte.162.10.1144

6. Labropoulos N, Webb KM, Kang SS, Mansour MA, Filliung DR, Size GP, Buckman J, Baker WH. Patterns and distribution of isolated calf deep vein thrombosis. J Vasc Surg. 1999;30(5):787-91. https://doi.org/10.1016/s07415214(99)70002-9 PMID: 10550175.

7. Yao Y, Liang Q, Kai S, Xingquan X, Dongquan S, Zhihong X, et al. Preoperative evaluation of soleal vein diameter by ultrasound is beneficial for prophylaxis of deep vein thrombosis after total knee or hip arthroplasty. BioMed Res Int. 2018;1-8. https://doi.org/10.1155/2018/3417648

8. Abe K, Yuda S, Yasui K, Okubo A, Kobayashi C, Muranaka A, et al. Soleal vein dilatation assessed by ultrasonography is an independent predictor for deep vein thrombosis after major orthopedic surgery. J Cardiol. 2016; 9:75662. https://doi.org/10.1016/j.jjcc.2016.07.004

9. Ashwin S, Mark D, Timothy L. Graduated compression stockings for prevention of deep vein thrombosis. Cochrane Data Syst rRv. 2018;11: CD001484.

10. Agu O, Hamilton G, Baker D. Graduated compression stockings in the prevention of venous thromboembolism. Br J Surg. 1999;86(8): 992-1004. https://doi.org/10.1001/archinte.1994.00420010095011

11. Maclellan DG, Fletcher JP. Mechanical compression in the prophylaxis of venous thromboembolism. ANZ J Surg. 2007;77(6):418-23. https://doi.org/1 0.1111/j.1445-2197.2007.04085.x.

12. Ayhan H, lyigun $E$, Ince $S$, Can MF, Hatipoglu S, Saglam M. A randomised clinical trial comparing the patient comfort and efficacy of three different graduated compression stockings in the prevention of postoperative deep vein thrombosis. J Clin Nurs. 2015;24(15-16): 2247-57. https://doi.org/1 $0.1111 /$ jocn.12866

13. Lord RSA, Hamilton D. Graduated compression stockings (20-30mmhg) do not compress leg veins in the standing position. ANZ J Surg. 2004;74(7). https://doi.org/10.1111/j.1445-2197.2004.02994.x.

14. Arcelus Jl, Caprini JA, Traverso Cl, Size G, Hasty JH. The role of elastic compression stockings in prevention of venous dilatation induced by a reverse trendelenburg position. Phlebology 1993;8(3): 111-5. https://doi. org/10.1177/026835559300800305

15. Smith C, Hasty JH, Scurr JH. Deep vein thrombosis: effect of graduated compression stockings on distension of the deep veins of the calf. $\mathrm{Br} J$ Surg. 2010;78(6). https://doi.org/10.1002/bjs.1800780628

16. Jeanneret C, Karatolios K, Planta I. Impact of compression stockings on calfvein diameters and on quality of life parameters in subjects with painful legs. Vasa Ztschrift Für Gefsskrankhten. 2014;43(4):268. https://doi.org/10.1 024/0301-1526/a000362.

17. Downie SP, Firmin DN, Wood NB, Thom SA, Hughes AD, Wolfe JNH, et al. Role of mri in investigating the effects of elastic compression stockings on the deformation of the superficial and deep veins in the lower leg. J Magn Reson Imag. 2010;26(1):80-5. https://doi.org/10.1002/jmri.20980.

18. Uhl JF, Benigni JP, Cornu-Thenard A, Fournier J, Blin E. Relationship between medical compression and intramuscular pressure as an explanation of a compression paradox. Phlebol J Venous Dis. 2015;30(5): 331-8. https://doi. org/10.1177/0268355514527442

19. Partsch $H$, Mosti G, Mosti F. Narrowing of leg veins under compression demonstrated by magnetic resonance imaging (mri). Int Angiol. 2010;29(5): 408. https://doi.org/10.1038/hr.2010.158. 
20. Giron FM, Mayberry JC, Moneta GL, Frang RDD. The influence of elastic compression stockings on deep venous hemodynamics. J Vas Surg. 1991; 13(1):91-100. https://doi.org/10.1067/mva.1991.25386.

21. Toya K, Takahashi T, Fujimoto Y. Effect of elastic stockings and ankle positions on the blood velocity in the common femoral vein. J Phys Ther Sci. 2016; 28(9):2608-10. https://doi.org/10.1589/jpts.28.2608

22. Stein PD, Matta F, Akkad MW, Hoppe CL, Patel YR, Sivakumar S. Effect of graduated compression stockings on venous blood velocity in supine resting hospitalized patients. Clin Appl Thrombosis. 2014;20(7):693-97. https://doi.org/10.1177/1076029613479821.

23. Stein PD, Matta F, Yaekoub AY, Ahsan ST, Badshah A, Younas F, et al. Effect of compression stockings on venous blood velocity and blood flow. Thromb Haemo. 2010;104(1):138-44. https://doi.org/10.1160/TH09-06-0365.

24. Keith SL, Mclaughlin DJ Jr, Cardullo AF, Jones PA, Rohrer CE, et al. Do graduated compression stockings and pneumatic boots have an additive effect on the peak velocity of venous blood flow? Arch Surg. 1992;127(6): 727-30. https://doi.org/10.1001/archsurg.1992.01420060107016.

25. Jamieson R, Calderwood CJ, Greer IA. The effect of graduated compression stockings on blood velocity in the deep venous system of the lower limb in the postnatal period. Bjog. 2010;114(10):1292-4. https://doi.org/10.1111/j.14 71-0528.2007.01421.x.

26. Loïc $\mathrm{E}$, Lapole $\mathrm{T}$. Effects of graduated compression stockings, local vibration and their combination on popliteal venous blood velocity. Phlebology. 2020;23:15-20. https://doi.org/10.1177/0268355520902000.

27. Cohen J. A power primer. Psychol Bull. 1992;112(1):155-9. https://doi.org/1 0.1037//0033-2909.112.1.155

\section{Publisher's Note}

Springer Nature remains neutral with regard to jurisdictional claims in published maps and institutional affiliations.

Ready to submit your research? Choose BMC and benefit from:

- fast, convenient online submission

- thorough peer review by experienced researchers in your field

- rapid publication on acceptance

- support for research data, including large and complex data types

- gold Open Access which fosters wider collaboration and increased citations

- maximum visibility for your research: over $100 \mathrm{M}$ website views per year

At $\mathrm{BMC}$, research is always in progress.

Learn more biomedcentral.com/submissions 